\title{
THE ORIGIN OF THE COSMIC GAMMA-RAY BACKGROUND IN THE MeV RANGE
}

\author{
Pilar Ruiz-Lapuente ${ }^{1,2,3}$, Lih-Sin The ${ }^{4}$, Dieter H. Hartmann ${ }^{4}$, Marco Ajello ${ }^{4}$, Ramon Canal ${ }^{3,5}$, Friedrich K. Röpke ${ }^{6,7}$, \\ Sebastian T. Ohlmann ${ }^{6}$, and Wolfgang HillebrandT ${ }^{2}$ \\ ${ }^{1}$ Instituto de Física Fundamental, Consejo Superior de Investigaciones Científicas, c/. Serrano 121, E-28006, Madrid, Spain \\ ${ }^{2}$ Max-Planck-Institut für Astrophysik, Karl-Schwarzschild-Str. 1, D-85748 Garching bei München, Germany \\ ${ }^{3}$ Institut de Ciències del Cosmos (UB-IEEC), c/. Martí i Franqués 1, E-08028, Barcelona, Spain \\ ${ }^{4}$ Department of Physics and Astronomy, Clemson University, Clemson, SC 29634, USA \\ ${ }^{5}$ Departament d'Astronomia i Meteorologia, Universitat de Barcelona, c/. Martí i Franqués 1, E-08028 Barcelona, Spain \\ ${ }_{7}^{6}$ Institute of Theoretical Physics and Astrophysics, University of Würzburg, D-97074, Würzburg, Germany \\ ${ }^{7}$ Heidelberg Institute for Theoretical Studies, Schloss-Wolfsbrunnerweg 35, D-69118, Heidelberg, Germany \\ Received 2015 February 20; accepted 2016 February 9; published 2016 March 30
}

\begin{abstract}
There has been much debate about the origin of the diffuse $\gamma$-ray background in the $\mathrm{MeV}$ range. At lower energies, AGNs and Seyfert galaxies can explain the background, but not above $\simeq 0.3 \mathrm{MeV}$. Beyond $\sim 10 \mathrm{MeV}$ blazars appear to account for the flux observed. That leaves an unexplained gap for which different candidates have been proposed, including annihilations of WIMPS. One candidate is Type Ia supernovae (SNe Ia). Early studies concluded that they were able to account for the $\gamma$-ray background in the gap, while later work attributed a significantly lower contribution to them. All those estimates were based on SN Ia explosion models that did not reflect the full 3D hydrodynamics of SN Ia explosions. In addition, new measurements obtained since 2010 have provided new, direct estimates of high- $z$ SN Ia rates beyond $z \sim 2$. We take into account these new advances to see the predicted contribution to the gamma-ray background. We use here a wide variety of explosion models and a plethora of new measurements of SN Ia rates. SNe Ia still fall short of the observed background. Only for a fit, which would imply $150 \%$ systematic error in detecting SN Ia events, do the theoretical predictions approach the observed fluxes. This fit is, however, at odds at the highest redshifts with recent SN Ia rate estimates. Other astrophysical sources such as flat-spectrum radio quasars do match the observed flux levels in the MeV regime, while SNe Ia make up to $30 \%-50 \%$ of the observed flux.
\end{abstract}

Key words: galaxies: active - gamma rays: diffuse background - supernovae: general

\section{INTRODUCTION}

The cosmic gamma-ray background is diffuse, and its origin is diverse and remains partly unknown at various energy ranges. On the low-energy side, from $\mathrm{X}$-ray energies up to around $0.3 \mathrm{MeV}$, AGNs and Seyfert galaxies provide most of the emission (Madau et al. 1994; Ueda et al. 2003). At $E \gtrsim 0.3 \mathrm{MeV}$, the spectrum of AGNs and Seyfert galaxies sharply cuts off. From $50 \mathrm{MeV}$ to the $\mathrm{GeV}$ range, blazars seem to be responsible for the observed flux (Zdziarski 1996; Sreekumar et al. 1998). However, the latest results from Fermi, in the $\mathrm{GeV}$ range, which show a higher gamma-ray background at $\mathrm{GeV}$ energies than previous results from EGRET, have called into question the former attribution to blazars as the main source (see discussions in Lacki et al. 2014). Nevertheless, recent examinations of this issue find that at energies $>100 \mathrm{MeV}$, blazars account for $\sim 50 \%$ of the background, while the other half is contributed by star-forming galaxies and radio galaxies (Ajello et al. 2015; Di Mauro \& Donato 2015).

The measurements in the $\mathrm{MeV}$ range have been provided by various space missions. The first exploration of the region between 1 and $5 \mathrm{MeV}$ was made by the APOLLO 15/16 missions (Trombka et al. 1977). The reanalysis of the Apollo data, the measurements from HEAO-A4 (Kinzer et al. 1997), the Solar Maximum Mission (SMM; Watanabe et al. 1999b), and COMPTEL (Kappadath et al. 1996; Weidenspointner 1999; Weidenspointner et al. 2000) provided the basic empirical results on the diffuse gamma-ray background, in the range from $100 \mathrm{keV}$ to $10 \mathrm{MeV}$. The slope of the emission spectrum exhibits a steep decrease with increasing energy, from a few hundred $\mathrm{keV}$ to $10 \mathrm{MeV}$, changing to a flatter slope around $10 \mathrm{MeV}$ and beyond, revealing the need of an intense extragalactic source in the $\mathrm{MeV}$ window. In this range of energies, the discussion of the origin of the background was revived at the beginning of the twenty-first century and continued up to the present time (see Lacki et al. 2014).

Much of the current discussion centers on the possibility that Type Ia supernovae (SNe Ia) are able to produce the observed flux in the $0.3-3 \mathrm{MeV}$ range, filling the gap between Seyferts and blazars. This possibility was first suggested by Clayton \& Silk (1969) and further studied by The et al. (1993) and Watanabe et al. (1999a, hereafter W99b). At those times, there were no empirical rates of SNe Ia available, in particular, the function $R_{I a}(z)$ was not measured at the required high redshifts. Therefore, the authors had to estimate fluxes on the basis of the better-known star formation rates in galaxies and a convolution with an assumed delay time distribution (DTD) between the birth of the progenitor binary systems and the explosions. Depending on the delay time since birth of the system, the SN Ia rate could be either too low or just right, to account for the measured level of the gamma-ray background (W99b). Ruiz-Lapuente et al. (2001, hereafter RCV01) performed a study of the SN Ia contribution in the $\mathrm{MeV}$ region, by considering a wide range of star formation rates $\dot{\rho}_{*}(z)$ in galaxies and different efficiencies in the production of SNe Ia, for each star formation rate. Ruiz-Lapuente (2001) found that not much under $1000 M_{\odot}$ going into star formation gives rise to one SN Ia (720 $\pm 250 M_{\odot}$ produce one SN Ia). The efficiency, 
defined as

$$
\mathcal{E}_{I a}(z)=\frac{R_{I a}(z) \mathrm{yr}^{-1} \mathrm{Mpc}^{-3}}{\dot{\rho}_{*}(z) M_{\odot} \mathrm{yr}^{-1} \mathrm{Mpc}^{-3}},
$$

was $(1.41 \pm 0.35) \times 10^{-3} M_{\odot}^{-1} h_{65}^{2}$. In that work, they found that the delay time between star formation and $\mathrm{SN}$ explosion could shift the estimate up or down, but that most star formation histories would average out the effect, since the distribution of SN Ia delay times appeared to be broad. The result of this study was that $\mathrm{SNe}$ Ia yield a background emission, in the $\mathrm{MeV}$ range, that can explain the extragalactic emission measured by COMPTEL and SMM.

Just shortly afterward, the topic was addressed by other authors (Iwabuchi \& Kumagai 2001; Ahn et al. 2005, hereafter AKH05; Strigari et al. 2005), and it was more recently considered again by Horiuchi \& Beacom (2010, hereafter HB2010). The latter authors obtained a range of possibilities concerning the SN Ia contribution, but found that they are in general not able to account for the $\mathrm{MeV}$ background. The earlier "negative" results can be traced to the first high- $z$ SN Ia rates obtained by Dahlen et al. (2004, hereafter D04), which peak at a lower $z$ than current rates (Graur et al. 2014; Okumura et al. 2014; Rodney et al. 2014). However, HB2010 do not use the Dahlen et al. (2004) rates, but a common fit to data availablle up to 2010 . These rates will be compared with most recent measurements.

Both W99b and RCV01 (see also similar findings by The et al. 1993; Zdziarski 1996) had concluded that there was room for SNe Ia to account for the unexplained $\mathrm{MeV}$ background. They did so by integrating the full gamma-ray spectrum emerging from SN Ia models during the early period of significant production by radioactive decay. In this context one includes the production of gamma-ray photons, as well as their transport in the expanding ejecta. AKH05 take an SN Ia rate (from D04) that is one order of magnitude smaller than the one adopted by W99b. This may explain most of the discrepancy in the results. HB2010 calculations take the continuum plus line $\gamma$-ray spectra from the W7fm model (fully mixed W7 model) shown in W99b and the 5p0z22.23 model shown in AKH05 (private communication). We find agreement with their results when using the same SN Ia rates as HB2010 did.

One interesting feature of the SN Ia contribution, which is shared by the calculations presented in W99b, RCV01, AKH05, HB2010, is that the predicted SN Ia gamma-ray background contribution has a spectral shape (power law in energy) that runs parallel to the observed fluxes up to $3 \mathrm{MeV}$. This match in slope is one of the primary reasons for considering $\mathrm{SNe}$ Ia as a significant contributor and for focusing on the disagreement with the flux normalization.

Owing to the recent appreciation of SNe Ia as cosmological probes, a significant increase in dedicated observational programs produced a greatly improved knowledge of their rates, for a wide range of redshifts (some of the most recent contributions were mentioned above). We also know well the cosmological parameters required for the calculation $\left(H_{0}, \Omega_{M}\right.$, $\left.\Omega_{\Lambda}\right)$. Moreover, for the first time, a nearby SN Ia (SN 2014J) has been detected in the $\mathrm{MeV}$ range (with INTEGRAL; Churazov et al. 2014; Diehl et al. 2014). The latter authors account for the gamma-ray data with a white dwarf (WD) explosion having a small amount of ${ }^{56} \mathrm{Ni}$ at the outskirts and around $0.5 M_{\odot}$ in the innermost core. This model is similar to those used in the previous calculations of the gamma-ray background (and also to those that will be used here). Thus, we now have a firmed-up grasp of the gamma-ray flux emitted by $\mathrm{SNe}$ Ia.

These developments led us to once more calculate the gamma-ray background from SNe Ia, now based on improved knowledge of the theoretical ingredients. This paper is organized as follows. In Section 2, we discuss the different numerical methods used to compute the gamma-ray fluxes from given SN Ia models. The models used for the present study are described in Section 3. In Section 4, we present an update of the $\mathrm{SN}$ Ia rates at various $z$ and show the interpolations used for the background calculations. In Section 5, we explain the gamma-ray background formulation and present the results. Section 6 discusses the results, and the final section contains our conclusions.

\section{GAMMA-RAY ESCAPE}

In order to derive the emergent spectrum predicted by SN models, we use the Monte Carlo code described in Burrows \& The (1990) and The et al. (1990), modified to include bremsstrahlung X-ray production and the iron fluorescence line at $\sim 6.4 \mathrm{keV}$ (Clayton \& The 1991; The et al. 1994). This code follows the gamma-ray emission of radioactive nuclei such as ${ }^{44} \mathrm{Ti},{ }^{56} \mathrm{Ni},{ }^{56} \mathrm{Co}$, and ${ }^{57} \mathrm{Co}$. The code was used in the predictions by W99b.

In RCV01, a modified version of the Ambwani \& Sutherland (1998) code was used, which was also employed by Lehoucq et al. (1989) to study the radioactive output of SN 1987A. It was further modified (Ruiz-Lapuente et al. 1993) to include positronium formation in the ${ }^{56} \mathrm{Co}$ decay, giving rise to twophoton (parapositronium) and three-photon decay (orthopositronium), the energy distribution being that derived by Bussard et al. (1979). The Monte Carlo routine was then used for the study of gamma rays from SNe Ia (Ruiz-Lapuente et al. 1993). A comparison of the predicted gamma-ray emission, for model W7 (Nomoto et al. 1984), with that from W99b shows the gamma-ray fluxes predicted from the code used in RCV01 to be $10 \%$ higher in the $\mathrm{MeV}$ regime than those from W99b. Differences of the gamma-ray yields resulting from different gamma-ray transport codes were studied in detail by Milne et al. (2004), who show that variations of $\sim 10 \%$ can arise from the different physics and methods employed. With the use of the same code as in W99b, Burrows \& The (1990), and The et al. (1990), we are on the conservative side concerning the predicted gamma-ray emission (see Milne et al. 2004).

\section{INPUT MODELS}

A wider range of predicted emerging fluxes in the $\mathrm{MeV}$ domain is obtained when using different input models for the SNe Ia, as recently shown by The \& Burrows (2014). Looking at these results, one notices that there are $\sim 20 \%$ variations, in the $\mathrm{MeV}$ range, between various input models. Here we pick five different models in an attempt to delineate the upper limit constraint on the gamma-ray emision from normal SNe Ia. These models are W7 (Nomoto et al. 1984), the fully mixed W7 model (W7fm) used in W99b (both models synthesize $0.58 M_{\odot}$ of ${ }^{56} \mathrm{Ni}$ ), the W7dt model of Yamaoka et al. (1992) (this one predicts a larger escaping gamma-ray flux than most of the other models explored by The \& Burrows [2014] as it synthesizes $0.78 M_{\odot}$ of ${ }^{56} \mathrm{Ni}$, a quantity slightly larger than in a 


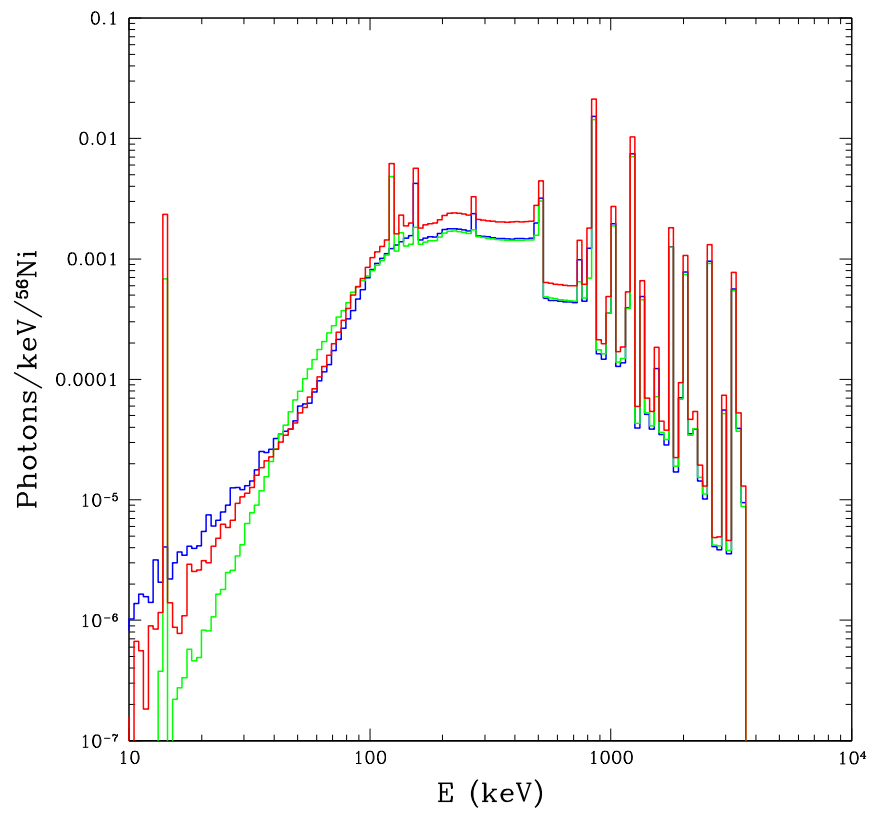

Figure 1. Total X-ray continuum and gamma-ray line fluxes per ${ }^{56} \mathrm{Ni}$ nuclei, integrated over the first 600 days. The green line shows the corresponding fluxes from the calculations of The et al. (1993) for the W7 model (Nomoto et al. 1984), the blue line shows the fluxes for the fully mixed W7 model (W7fm) used in W99b, and the red line shows the fluxes for the W7dt model from Yamaoka et al. (1992; see also The \& Burrows 2014).

normal SN Ia), the spherically averaged 3D model N100 of a delayed detonation (Röpke et al. 2012; Seitenzahl et al. 2013), and the (also averaged) 3D model of the violent merging of two WDs of Pakmor et al. (2012). Those two 3D averaged models synthesize, respectively, $0.604 M_{\odot}$ of ${ }^{56} \mathrm{Ni}$ in the model N100 and $0.62 M_{\odot}$ in the averaged $3 \mathrm{D}$ violent merging of two WDs, quantities within the canonical range of $0.55-0.65 M_{\odot}$ of ${ }^{56} \mathrm{Ni}$ for normal SNe Ia.

The first three models have been widely discussed in the literature. Their gamma-ray emissions are compared in Figure 1. The 3D model N100 of Röpke et al. (2012) corresponds to an initially isothermal WD, made of $\mathrm{C}$ and $\mathrm{O}$ in equal parts, with a central density of $2.9 \times 10^{9} \mathrm{~g} \mathrm{~cm}^{-3}$. It was ignited in 100 sparks (hence its name), placed randomly in a Gaussian distribution within a radius of $150 \mathrm{~km}$ from the WD's center. After an initial deflagration phase, a detonation was triggered at every location of the flame where the criterion for a deflagration-to-detonation transition of Ciaraldi-Schoolmann et al. (2013) was met.

The model from Pakmor et al. (2012), also a 3D model, simulates the violent merger of a $1.1 M_{\odot}$ WD and a $0.9 M_{\odot}$ WD. As the material of the tidally disrupted secondary WD hits the primary WD, a hot spot forms, which leads to the ignition of $\mathrm{C}$ burning. The conditions reached there are sufficient to trigger a detonation that burns the merged object and leads to the explosion.

The spectral evolution of the gamma-ray emission from the two 3D models above is shown in Figure 1 of Summa et al. (2013), together with the spread due to different viewing angles for the maximum-light epochs in gamma rays of both models. Here, however, we use the spherically averaged models, with the same treatment of gamma-ray escape as for the three 1D models (W7, W7fm, and W7dt). Their comparison with W7fm is shown in Figure 2.

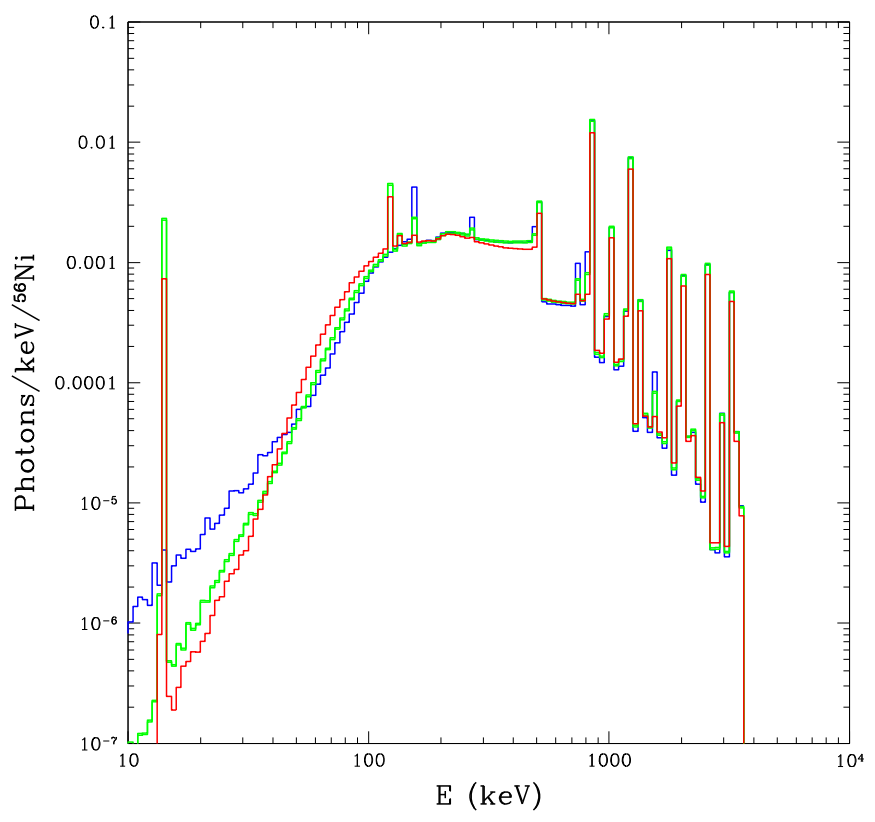

Figure 2. Total X-ray continuum and gamma-ray line fluxes per ${ }^{56} \mathrm{Ni}$ nuclei, integrated over the first 600 days comparing the calculations for the fully mixed W7 model (W7fm) used in W99b (blue line), for the (spherically averaged) delayed-detonation 3D model N100 of Röpke et al. (2012; green line), and for the (also averaged) 3D model of the violent merging of two WDs of Pakmor et al. (2012; red line).

Ruiter et al. (2013) have shown that sub-Chandrasekhar pure detonation models can reproduce the observed peak-magnitude distribution of SNe Ia ( $\mathrm{Li}$ et al. 2011b). The brightness of the explosion is mainly determined by the ${ }^{56} \mathrm{Ni}$ mass synthesized in the detonation of the primary WD. Sim et al. (2010) derived a relationship between the mass of that WD and the expected peak bolometric brightness. Ruiter et al. (2013) use the population synthesis data from Ruiter et al. (2011) to derive the theoretical peak-magnitude distribution.

Here we estimate the contribution of this channel to the gamma-ray background, based on the fact that the distributions of $\mathrm{WD}$ and ${ }^{56} \mathrm{Ni}$ masses peak at 1.1 and $0.6 M_{\odot}$, respectively. In Figure 3 we compare the gamma-ray spectrum of a representative model of such mergings with that of model W7fm. The major difference between models is in the X-ray domain between 1 and $50 \mathrm{keV}$. However, in the range of $\mathrm{MeV}$, the difference between these models that reproduce the bulk of $\mathrm{SNe}$ Ia in the optical is of order $5 \%$. Figure 4 shows the difference between the models in different energy ranges.

What has been shown in this section is that the background arising from different explosion models, associated with different progenitor origin channels (but all aiming at explaining normal SNe Ia), induces only a small variance. The convolution of the gamma-ray yield resulting from several models, here exemplified by model $\mathrm{W} 7 \mathrm{fm}$, with the $\mathrm{SN}$ rates is explored in Section 5.

\section{SN IA RATES}

Several groups have obtained SN Ia event rates using either ground-based facilities, the combination of ground-based and space facilities such as the Subaru/XMM-Newton Deep Survey (Okumura et al. [2014] being the most recently published search of this kind), or the Hubble Space Telescope (Rodney et al. 2014; Graur et al. [2014] have presented the most recent 


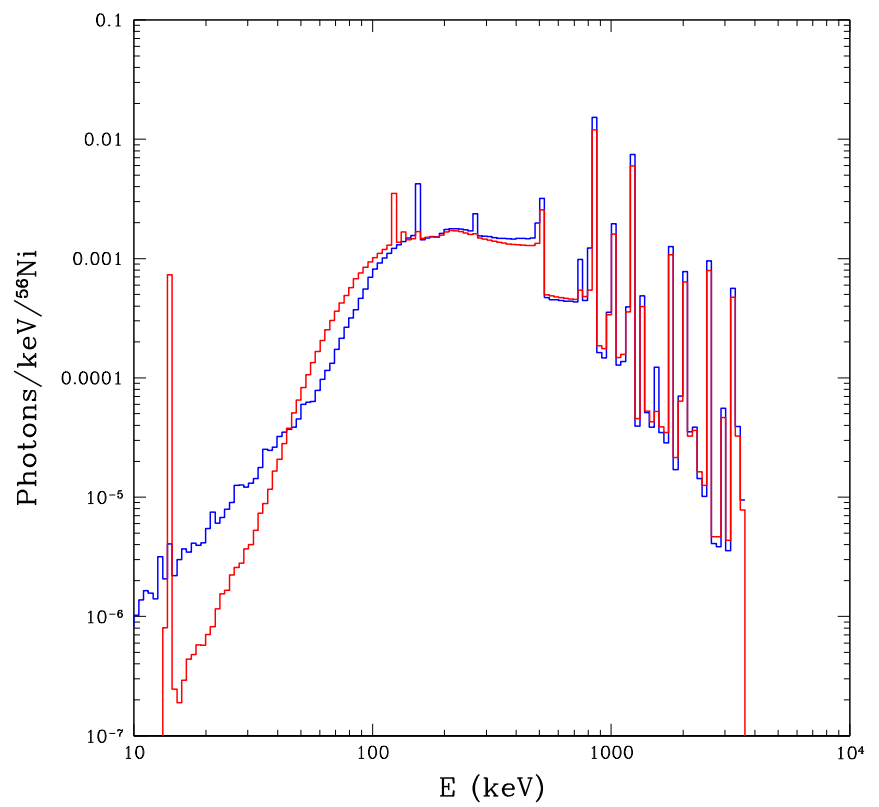

Figure 3. Total time-integrated X-ray continuum and gamma-ray line fluxes per ${ }^{56} \mathrm{Ni}$ nucleus, comparing the calculations for an average model of the subChandrasekhar pure detonation models (Sim et al. 2010; Ruiter et al. 2013; red line) and for the W7fm model of W99b (blue line).

results from such searches). One goal of those surveys is to gauge the DTD of SNe Ia, i.e., the time that the SN Ia progenitors spend between formation and explosion.

Figure 5 shows how the different fits to compilations by Okumura et al. (2014), Graur et al. (2014), and Rodney et al. (2014) pass through the data up to $z \sim 1$ with similar lines, but they differ significantly at larger redshifts. For a close look at low and high $z$, we plot in Figure 6 all measurements made up to now, taken from the compilation in Table 4 of Graur et al. (2014) (superseded ones excluded). ${ }^{8}$

The estimates of the SN Ia rates are derived in different ways. Okumura et al. (2014), following previous work by the Supernova Cosmology Project, select objects having a flux larger than $5 \sigma_{b}$, where $\sigma_{b}$ is the background fluctuation within an aperture of 2 arcsec in diameter. These objects are classified as transients. The authors require that objects show at least a $5 \sigma_{b}$ increase in two or more epochs, in the $i^{\prime}$ band. This requirement is accounted for in the rate calculation when computing the control time. For the SN hosts for which spectroscopic redshifts are not available, they use photometric redshifts from the probability distribution function of the host galaxy redshifts. They discriminate against AGNs using X-ray

\footnotetext{
8 In order of increasing redshift, they are from Cappellaro et al. (1999), Li et al. (2011a), Dilday et al. (2010), Dilday et al. (2008), Dilday et al. (2010), Graur \& Maoz (2013), Blanc et al. (2004), Hardin et al. (2000), Dilday et al. (2010), Rodney \& Tonry (2010), Perrett et al. (2012), Horesh et al. (2008), Dilday et al. (2010), Dilday et al. (2010), Perrett et al. (2012), Botticella et al. (2008), Dilday et al. (2010), Rodney \& Tonry (2010), Perrett et al. (2012), Graur et al. (2014), Rodney \& Tonry (2010), Perrett et al. (2012), Graur et al. (2014), Rodney \& Tonry (2010), Perrett et al. (2012), Tonry et al. (2003), Dahlen et al. (2008), Pain et al. (2002), Rodney \& Tonry (2010), Perrett et al. (2012), Melinder et al. (2012), Rodney \& Tonry (2010), Perrett et al. (2012), Graur et al. (2011), Rodney \& Tonry (2010), Perrett et al. (2012), Barbary et al. (2012), Dahlen et al. (2008), Rodney \& Tonry (2010), Perrett et al. (2012), Graur et al. (2014), Rodney \& Tonry (2010), Perrett et al. (2012), Rodney \& Tonry (2010), Perrett et al. (2012), Barbary et al. (2012), Dahlen et al. (2008), Graur et al. (2011), Barbary et al. (2012), Graur et al. (2014), Dahlen et al. (2008), Graur et al. (2014), Dahlen et al. (2008), and Graur et al. (2011) and are shown as open circles in the figure.
}

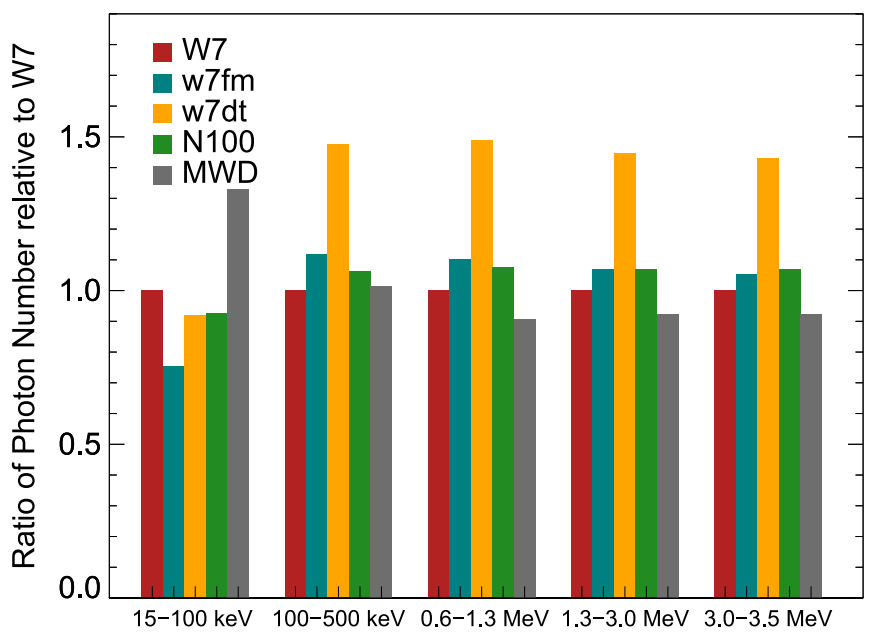

Figure 4. Ratio of the number of emergent photons relative to model W7. The bar graph shows the ratio of the integrated number of emergent photons of models considered here relative to W7. Note that photons in energy bands below $0.6 \mathrm{MeV}$ are not relevant to the cosmic gamma-ray background near$\mathrm{MeV}$ region, which is our main consideration, but we plot them for completeness. Please note the good agreement within $5 \%$ of the $3 \mathrm{D}$ models with the w7 fm model, as well as with the W7 model. The model w7dt has more ${ }^{56} \mathrm{Ni}$ mass than a normal SN Ia.

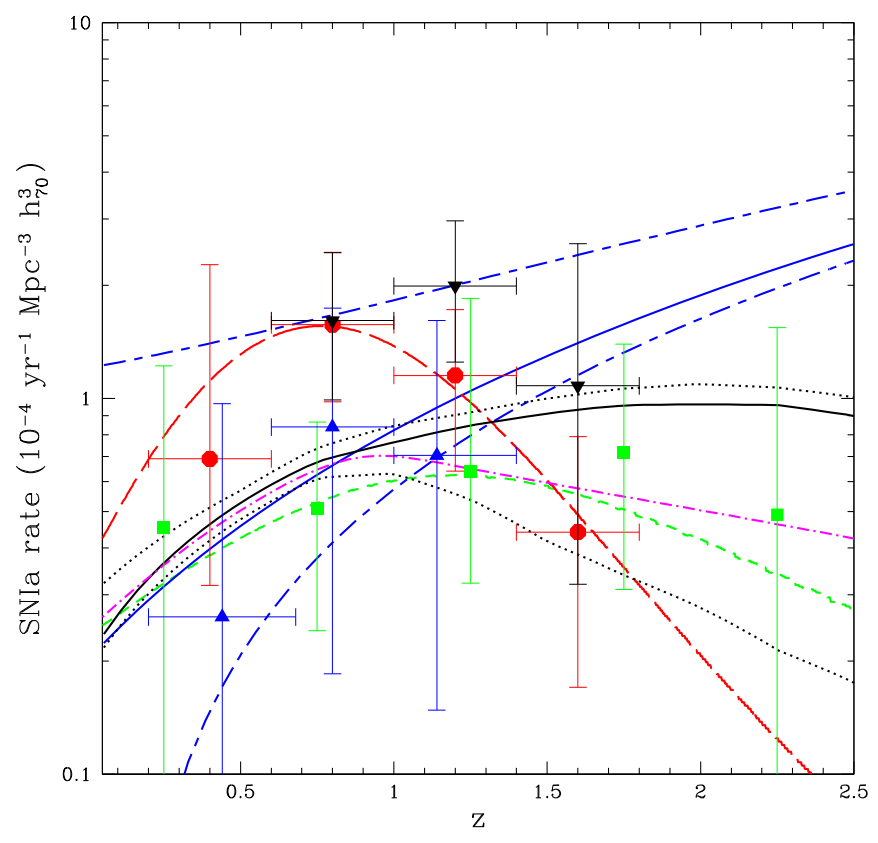

Figure 5. SN Ia rates measured at different redshifts and diverse fits to them. Red circles are D04 data from Dahlen et al. (2004); green squares come from the CANDELS program (Rodney et al. 2014); blue triangles are from the Subaru/XMM-Newton Deep Survey (Okumura et al. 2014); inverted black triangles correspond to the HST Cluster Supernova Survey (Barbary et al. 2012). The red long-dashed line is a fit to the D04 data, done as in AKH05. The green short-dashed line is the same fit to the CANDELS data shown in Figure 11 of Rodney et al. (2014), while the magenta dot-dashed line corresponds to the rates used by HB2010 (private communication). The solid black line is a fit to the rates given in Table 6 of Graur et al. (2014) (see next figure for details), and the two black dotted lines are its $\pm 1 \sigma$ limits. Finally, the blue solid line is the same fit as in Figure 11 of Okumura et al. (2014) to the Subaru/XMM-Newton Deep Survey data, and the two blue long-dashed-shortdashed lines its $\pm 1 \sigma$ limits. Note that the upper $1 \sigma$ limit just fits the two highest measured rates. The $\pm 1 \sigma$ regions around the rates from Okumura et al. (2014) have been traced by calculating the error using the statistical and systematic errors of the points measured by these authors. Note that the upper systematic errors include $50 \%$ extinction by dust, which explains why the upper error line is more distant from the main fit than the lower one. 


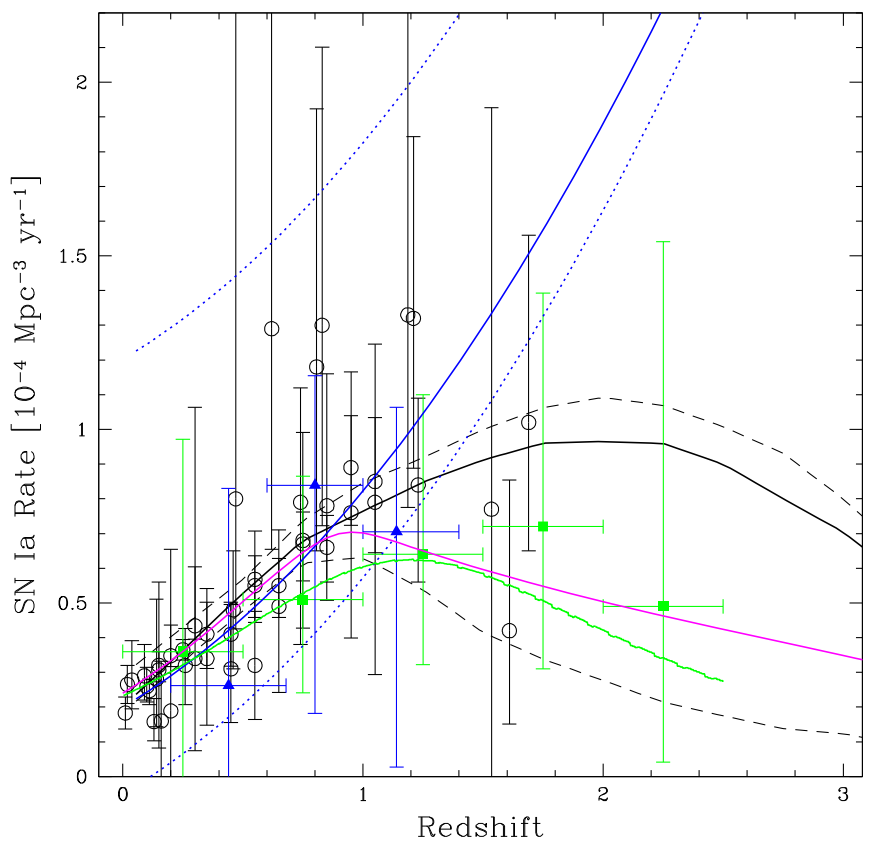

Figure 6. Different fits to the currently available SN Ia rate data, up to a redshift $z \simeq 2.25$. The solid black line is a fit to the rates given in Table 6 by Graur et al. (2014) and the two black dashed lines its $\pm 1 \sigma$ limits. The blue solid line running up to the upper edge of the diagram is the fit by Okumura et al. (2014) to the Subaru/XMM-Newton Deep Survey data, and the two dotted lines the $\pm 1 \sigma$ limits. The green solid line is the fit to the CANDELS data (shown as green squares) alone, from Rodney et al. (2014), while the magenta solid line corresponds to the SN Ia rates adopted by HB2010 (private communication). The open circles are the data from different surveys, covering from $z=0$ up to $z \simeq 1.7$ (see the text for the references). Note that, when comparing with the previous figure, the vertical scale is here linear instead of semilogarithmic. The $\pm 1 \sigma$ regions around the rates from Okumura et al. (2014) have been traced by calculating the error using the statistical and systematic errors of the points measured by these authors. Note that the upper systematic errors include $50 \%$ extinction by dust, which explains why the upper error line is more distant from the main fit than the lower one.

data from observations with XMM-Newton. Template light curves help to discriminate $\mathrm{SNe}$ Ia from $\mathrm{SNe} \mathrm{Ib} / \mathrm{II}$.

The final count of SNe Ia detected leads to the number of $\mathrm{SNe}$ per comoving volume unit, $r_{V}(z)$. The number of SNe Ia expected in a redshift bin $\left(z_{1}<z<z_{2}\right)$ is given by (Okumura et al. 2014)

$$
N_{\exp }\left(z_{1}<z<z_{2}\right)=\int_{z_{1}}^{z_{2}} r_{V}(z) \frac{C T(z)}{1+z} \frac{\Theta}{4 \pi} V(z) d z
$$

where $V(z) d z$ is the comoving volume in a redshift slice of thickness $d z, \Theta$ is the solid angle observed in the survey (in units of steradians), and $C T(z)$ is the observer frame "control time," i.e., the total time for which the survey is sensitive to an SN Ia at redshift $z$. The SN Ia rates per comoving volume of the Subaru/XMM-Newton Deep Survey (Okumura et al. 2014) are represented by blue triangles in Figures 5 and 6 together with the compilation of rates at all different $z$. They include a generous $50 \%$ error in the systematic uncertainties due to dust obscuration leading to lack of detection of $\mathrm{SNe}$ Ia. In the figures, we also plot the $1 \sigma$ upper and lower bounds of Okumura et al. (2014) rates as two blue long-dashed-short dashed lines. They represent the total, statistical and systematic, uncertainties of the points measured by Okumura et al. (2014).
Graur et al. (2014) present a compilation of all previous measurements of SN Ia rates and new rates obtained by the CLASH collaboration in the range $1.8<z<2.4$. The procedure they use to compute the rates has aspects in common with the Okumura et al. (2014) approach, as they look for candidates in this case with a flux $3 \sigma$ above the background and they rely on a good knowledge of the redshift of either the SN or its host galaxy. But it also includes some Bayesian approaches in the classification of the SN with a probability obtained by comparing the light curve of the SN with lightcurve models, as in Rodney et al. (2014).

Rodney et al. (2014) (for the CANDELS collaboration) use techniques and requirements different from those of Okumura et al. (2014). They use a Bayesian photometric classification algorithm based on the light curves of high-redshift SNe. The main uncertainties in this approach are the redshift-dependent prior describing the relative fraction of $\mathrm{SNe}$ that are $\mathrm{SNe}$ Ia and the assumed distribution of dust extinction values. The hostbased prior comes from the algorithm galsnid of Foley \& Mandel (2013), which exploits the relationships between SN and their host environments. They adopt a distribution of host galaxy extinctions for the dust extinction that then enters as a further probability into the SN Ia classification algorithm. The mid-rate plus mid-dust combination gives their baseline classification probability, which determines how much each individual SN contributes to the total count of observed SNe Ia. The upper and lower bounds make the error bars. The SN Ia rates from the CANDELS collaboration are plotted as green squares in Figures 5 and 6.

The utilization of informed Bayesian priors to estimate the SN Ia rate as a function of redshift was published earlier by Graur et al. (2011). Kuznetsova et al. (2008) had followed a Bayesian approach as well, to determine the rates in four redshift bins.

The most conservative $\mathrm{SN}$ Ia rate error bars derived up to date are those from Barbary et al. (2012). We show them as inverted black triangles in Figures 5 and 6 . The study considers different dust models in estimating SN Ia rates where it gives the highest estimates of the observed SN Ia rates and also the largest uncertainties. The large error bars also result from the large uncertainties of dust extinction estimates.

The D04 data are shown as red circles in Figure 5. The fit to these data appears as the red long-dashed line in the figure. As can be seen, we have fitted the D04 data in a way similar to that in AKH05. We have considered, as in that work, that the first error bar is reduced by taking the mean value of the upper and lower error bars of the first $z$.

One controversial aspect in the $1 \sigma$ and $2 \sigma$ error bars from Okumura et al. (2014) is the way they are calculated. If we were to take the $1 \sigma$ upper and lower limits to the SN Ia rates by propagating the errors in the fitting parameters given by their expressions (10)-(12), the upper $1 \sigma$ limit would be at odds with the data from all other surveys and also well beyond that indicated by the uncertainties of their own data. There is no questionable deduction, however, of the $1 \sigma$ errors of their individual measurements presented for the first time in their 2014 paper. Nevertheless, the extrapolation of the fitting formula beyond their data departs significantly from what has been found by other authors (Graur et al. 2014; Rodney et al. 2014). We keep their fitting formula, given that discrepancies start beyond the redshift range from within which most of the 
background arises, and we use the $1 \sigma$ and $2 \sigma$ uncertainties as derived from their data points.

To date there remain divergences in the measured SN Ia rates, despite the considerable increase of the database in recent years. They reflect the differences in the procedures followed to derive the rates from the observations. In particular, how the SN Ia rates are estimated accounts for dust obscuration.

The approach by Okumura et al. (2014) is well tested. It clearly discards SNe Ia from AGNs and SNe of other types. Contamination by $\mathrm{SNe} \mathrm{Ibc}$ is estimated and taken into account in the systematic uncertainty. These authors acknowledge the significant uncertainty related to dust extinction (50\%). Note the present disparity in the treatment of dust obscuration and the very different approaches taken by different authors.

We just report the published rates and their corresponding gamma-ray distributions based on three different estimates of these rates at the highest available $z$ to show the level of uncertainty at high $z$, while they are consistent at low $z$, where these three major estimates take into account all previously measured SN Ia rates (see Figures 5 and 6). We calculate the gamma-ray background contribution corresponding to those SN Ia rates.

\section{BACKGROUND CONTRIBUTIONS}

Based on the gamma-ray spectra in Figures 1-3 (number of escaping gamma photons per $\mathrm{keV}$ and per nucleus of ${ }^{56} \mathrm{Ni}$ synthesized in the explosion, as a function of energy $E$ ), calculated for different SN Ia models, we compute the average luminosity $l_{\gamma}(E)$ (photons $\mathrm{s}^{-1} \mathrm{keV}^{-1}$ ) of an SN Ia from the amount of ${ }^{56} \mathrm{Ni}$ in the model and the time along which the SN has been emitting. In the input spectra, all the photons emitted from the time of the explosion to 600 days later were collected. Therefore, the number of active SNe Ia per unit of comoving volume, at any time, is that of those produced during the preceding time interval: $R_{I a}^{\prime}(z)=$ const. $\times R_{I a}(z)$, the latter being the comoving SN Ia rate $\left(\mathrm{SNe} \mathrm{yr}^{-1} \mathrm{Mpc}^{-3}\right)$. Thus, the contribution to the gamma-ray background of the shell at comoving radius $r$ and with thickness $d r$ would be

$$
d L_{\gamma}(E, z)=4 \pi R_{I a}^{\prime}(z) l_{\gamma}(E) d V(z),
$$

where

$$
d V(z)=d_{M}^{2}(z) d\left(d_{M}\right)
$$

$d_{M}$ being the proper motion distance (in $\mathrm{Mpc}$ ). The flux received from that shell (in photons $\mathrm{cm}^{-2} \mathrm{~s}^{-1} \mathrm{keV}^{-1}$ ) will be

$$
d F_{\gamma}(E, z)=\frac{1}{4 \pi d_{L}(z)^{2}} d L_{\gamma}[(z+1) E, z]
$$

$d_{L}$ being the luminosity distance (in $\mathrm{cm}$ ). The factor $(z+1)$, multiplying $E$, accounts for the redshift of the photons. We thus have

$$
d F_{\gamma}(E, z)=R_{I a}^{\prime}(z) l_{\gamma}[(z+1) E] \frac{d_{M}^{2}(z)}{d_{L}^{2}(z)} d\left(d_{M}\right)
$$

Owing to time dilation, there should be a factor $(1+z)^{-1}$ multiplying the comoving $\mathrm{SN}$ rate, but it is canceled by the $(1+z)$ factor accounting for compression of the energy bins.

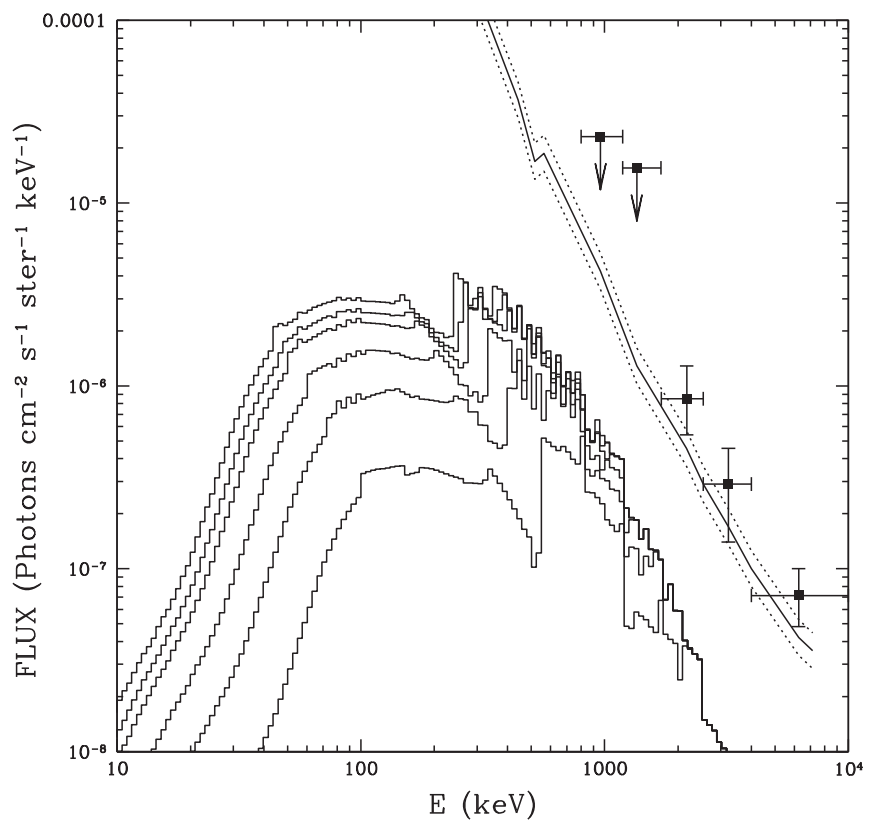

Figure 7. Contributions to the gamma-ray background when considering the mean value fit to the SN Ia rates from Subaru-XMM (Okumura et al. 2014), for redshifts up to $z=0.50,1.00,1.50,2.00,2.25$, and 2.50 (curves in the order of increasing backgrounds at energy $E$ around $100 \mathrm{keV}$ correspond to the also increasing values of $z$ ), for model W7fm. One can see that, after $z=2$, the additional contribution becomes very small. Here and in the next three figures, the black squares correspond to the COMPTEL data, analyzed by Kappadath et al. (1996), while the solid line gives the results from the Solar Maximum Mission (Watanabe et al. 1999b), the dotted lines being the $1 \sigma$ upper and lower limits.

Then, since $d_{L}=(1+z) d_{M}$,

$$
d F_{\gamma}(E, z)=\frac{1}{(1+z)^{2}} R_{I a}^{\prime}(z) l_{\gamma}[(z+1) E] d\left(d_{M}\right),
$$

$d\left(d_{M}\right)$ depends on the cosmological parameters $H_{0}, \Omega_{M}$, and $\Omega_{\Lambda}$, and

$$
\begin{aligned}
F_{\gamma}(E)= & \frac{c}{H_{0}} \int_{0}^{z_{\lim }} \frac{1}{(1+z)^{2}} R_{I a}^{\prime}(z) l_{\gamma}[(z+1) E] \\
& \times e\left(z, \Omega_{M}, \Omega_{\Lambda}\right) d z .
\end{aligned}
$$

We adopt $H_{0}=70 \mathrm{~km} \mathrm{~s}^{-1} \mathrm{Mpc}^{-1}, \Omega_{M}=0.3$, and $\Omega_{\Lambda}=0.7$ in calculating our cosmic gamma-ray background. Indeed, the adopted values for $\Omega_{M}$ and $\Omega_{\Lambda}$ are the values favored by the PLANCK collaboration (Ade et al. 2014). The $H_{0}=70 \mathrm{~km} \mathrm{~s}^{-1}$ $\mathrm{Mpc}^{-1}$ value is a mean value coming from the discussions of the various approaches to determine $H_{0}$.

Thus, the universe is flat and

$$
e\left(z, \Omega_{M}, \Omega_{\Lambda}\right)=\left[(1+z)^{2}\left(1+\Omega_{M} z\right)-z(2+z) \Omega_{\Lambda}\right]^{-1 / 2} .
$$

We take $z_{\lim }=2.5$, as our various test calculations show that the contribution to the background from $\mathrm{SNe}$ Ia at higher redshifts is negligible. That can readily be understood because SN Ia rates drop significantly at redshifts $z$ larger than $\sim 2$. Furthermore, in order to compare calculated fluxes with observed values, we need to divide the $F_{\gamma}(E)$ above by $4 \pi$, to convert to the units of observed fluxes (photons $\left.\mathrm{cm}^{-2} \mathrm{~s}^{-1} \mathrm{keV}^{-1} \mathrm{sr}^{-1}\right)$. 


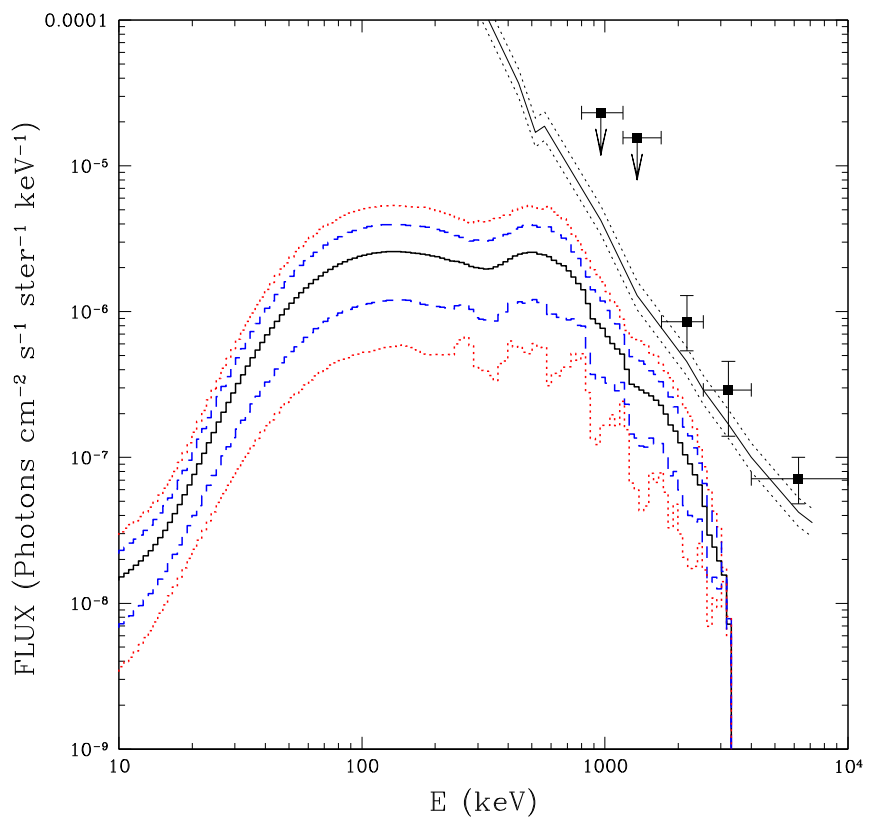

Figure 8. Gamma-ray background obtained using the mean fit from D04 to the SN Ia rates (see Figure 4) as the black solid lines and the $1 \sigma$ upper and lower bounds as blue dashed lines. The $2 \sigma$ upper and lower bounds are shown as the red dashed lines. With those rates, the SNe Ia cannot account for the observed gamma-ray background. We have used here the fully mixed W7 model (W7fm), as in all the other figures. Indeed, our results for the cosmic gammaray background shown here as the black solid line coincide with those of AKH05.

In Figure 7, we first show the calculated cosmic gamma-ray background from SN Ia model W7fm (W99b), for the SN Ia rates of Okumura et al. (2014). The rate is the main fit to the data of Okumura et al. (2014) at different redshifts $z$ (the blue solid line in Figures 5,6). We show the predicted backgrounds when considering the contributions from SNe Ia up to $z=0.50$, $1.00,1.50,2.00,2.25$, and 2.50 , respectively (the increasing contributions correspond to the also increasing values of $z$ ). It can be seen that, indeed, after $z=2$, further contributions to the background are negligible. In the figure, the black squares correspond to the COMPTEL data, analyzed by Kappadath et al. (1996), while the solid line gives the results from the Solar Maximum Mission (Watanabe et al. 1999b), the dotted lines being the $1 \sigma$ upper and lower limits.

In order to make a consistency check, we calculate the gamma-ray background from rates resulting from the fit to the D04 data (red dashed line in Figures 5 and 6) and its $1 \sigma$ and $2 \sigma$ predictions, using the W7fm model. Though the model is not the same as that used in AKH2005, we expect that our predictions should not significantly differ from theirs. Indeed, our resulting cosmic gamma-ray background, shown in Figure 8 as a black solid line, coincides with that of AKH05. In Figure 8 , we also plot the $1 \sigma$ upper limit as the blue dashed line and the $2 \sigma$ upper limit of the cosmic gamma-ray background using the D04 SN Ia rate as the red dashed line, confirming AKH05's conclusion that $\mathrm{SNe}$ Ia cannot account for the observed gamma-ray background. However, that conclusion is mostly a direct consquence of the D04 SN Ia rate used in the calculations, as we shall see below.

Having calculated the gamma-ray background using the fit of the SN Ia rate to the D04 data, we now consider the fit of Okumura et al. (2014). In Figure 9, we show the background

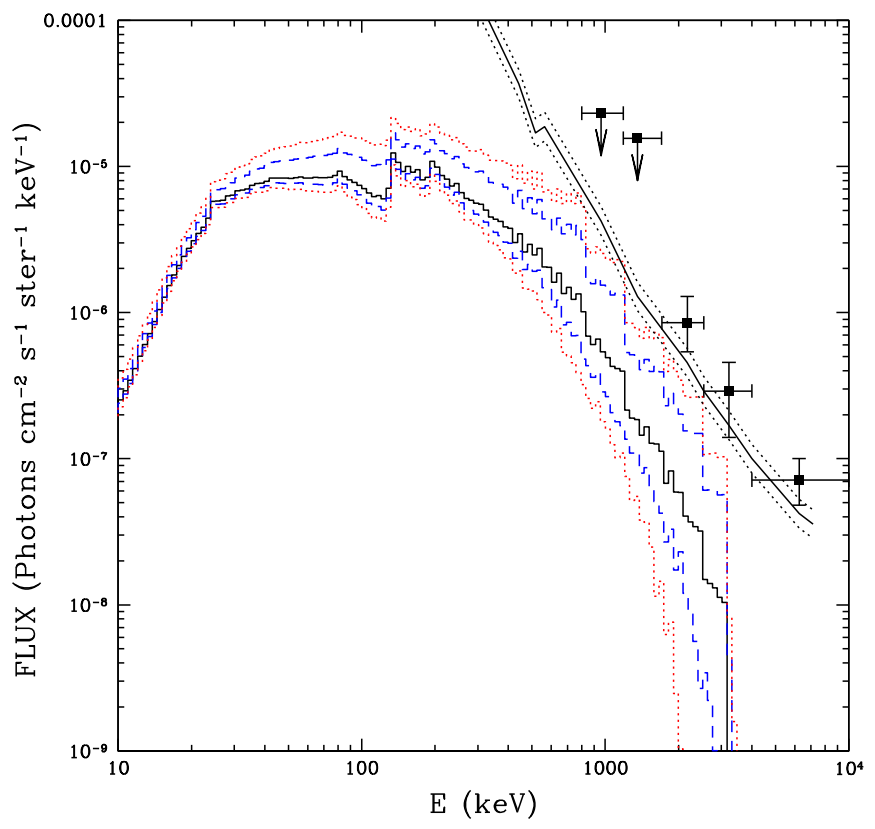

Figure 9. Gamma-ray background using the Okumura et al. (2014) fit to the observed SN Ia rates (black solid line), together with its $\pm 1 \sigma$ bounds (blue dashed lines) and $\pm 2 \sigma$ bounds (red lines). The $\pm 1 \sigma$ and $\pm 2 \sigma$ bounds have been traced by calculating the total error in the rates from the statistical and systematic errors of the points measured by these authors. The upper systematic errors include $50 \%$ extinction by dust. The model used is the fully mixed W7fm (W99b), but predictions for the models W7, W7dt, N100, and the two models of the violent merging of a couple of WDs are qualitative similar.

spectrum for the mean value of the fit by Okumura et al. (the blue solid line in Figures 5 and 6) as the black solid line. Clearly, the best fit to the SN Ia rate from the Subaru/XMMNewton Deep Survey does not produce good agreement with the observed cosmic gamma-ray background from SMM and COMPTEL (Figure 9). In Figure 9, we also plot the background spectrum for the $\pm 1 \sigma$ limits of Okumura et al. (2014) shown in Figures 5 and 6 and their correspondings $\pm 2 \sigma$ bounds. We include the corresponding predictions in Figure 9 for the gamma-ray background as they represent the most favorable case for $\mathrm{SNe} \mathrm{Ia}$ as sources in the $\mathrm{MeV}$ range.

In this work, for completeness, we calculate the background fluxes using the SN Ia rates derived from the fit to the rates compiled by Graur et al. (2014) (which include their own CLASH data; Figure 10) and by Rodney et al. (2014) (CANDELS; Figure 11). The Rodney et al. (2014) rates, shown as the green dot-dashed line in Figures 5 and 6, fall much lower than the Okumura et al. (2014) rates. And the corresponding gamma-ray background fluxes are lower than the fluxes produced by using the rates from Okumura et al. (2014) (see Figures 10, 11). HB2010 (Figure 12) prediction is shown here for the purpose of checking for consistency when reproducing their estimate. This estimate, based on more modest rates than those from the Okumura et al. (2014) fit, would not give enough background to explain the $\mathrm{MeV}$ emission either.

One might argue that the SN Ia rates toward higher redshifts could be underestimated if one does not take into account the distribution of SN Ia light-curve stretches, because that would disfavor the detection of small-stretch, lower-luminosity SNe. However, all the groups mentioned above have taken due account of this effect in modeling observed rates. 


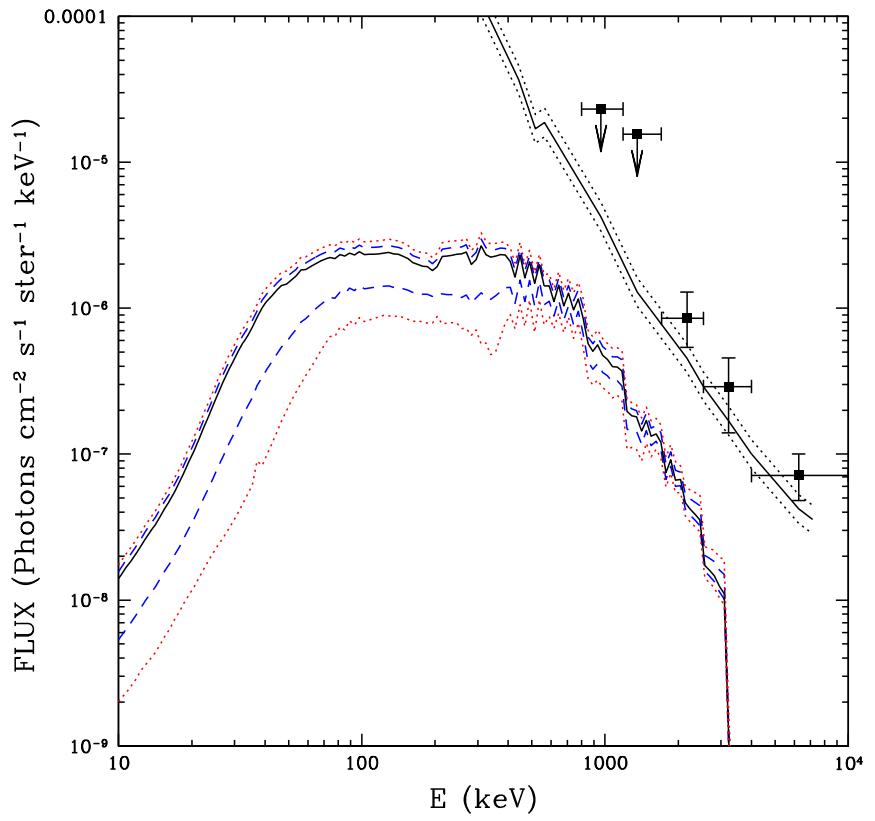

Figure 10. Gamma-ray background using the Graur et al. (2014) compilation of SN Ia rates (solid line; see Figures 5 and 6), and the upper $1 \sigma$ (blue dashed line) and $2 \sigma$ bounds (red dotted line). With those rates, the SNe Ia cannot account for the observed gamma-ray background at the $2 \sigma$ level (nor even at the $3 \sigma$ level). The model used is the fully mixed W7 (W99b), but predictions for the other models are qualitatively similar.

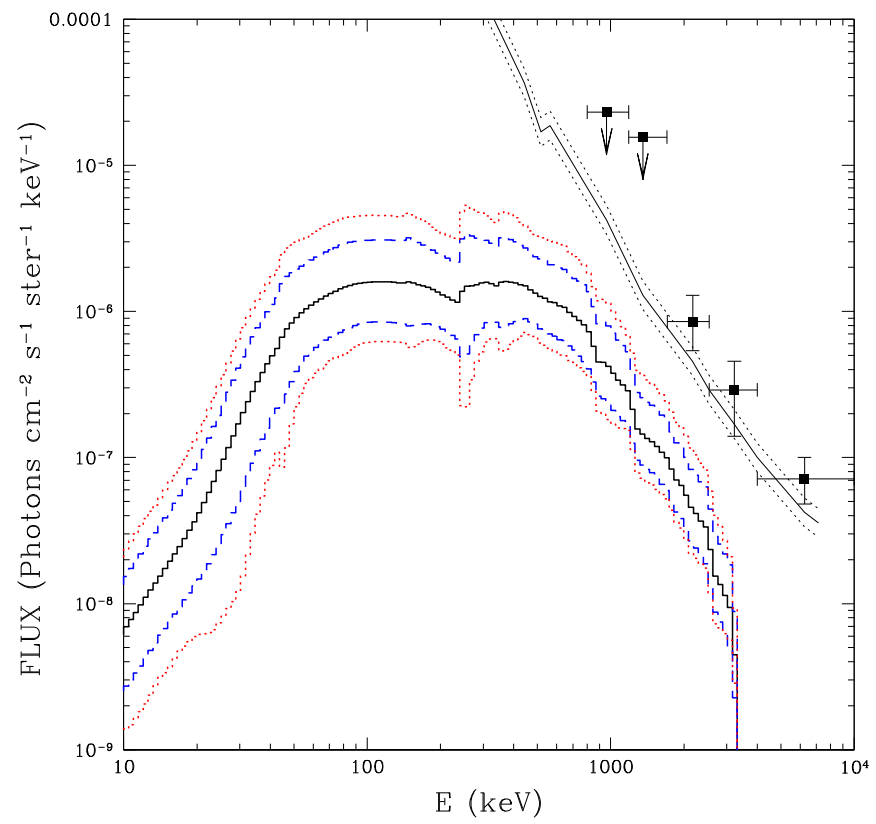

Figure 11. Gamma-ray background using the Rodney et al. (2014) (CANDELS) fit to the observational SN Ia rates with their prediction (solid black line) and the upper $1 \sigma$ (blue dashed line) and $2 \sigma$ bounds (red dotted line). With those rates, the SNe Ia cannot account for the observed gamma-ray background at the $2 \sigma$ level (nor even at the $3 \sigma$ level). The model used is the fully mixed W7 (W99b), but predictions for the other models are qualitatively similar.

\section{DISCUSSION}

$\mathrm{SNe}$ Ia are known to be prominent contributors to the annihilation line of positrons at $511 \mathrm{keV}$ and to the positronium continuum observed by $O S S E$ and by INTEGRAL. Milne et al. (2004) estimate that $30 \%-50 \%$ of Galactic positrons may be



Figure 12. Gamma-ray background using the HB2010 rates (private communication), together with their prediction (solid red line). The model used is the fully mixed W7 (W99b), but predictions for the other models are qualitatively similar. We show in red the calculations provided by HB2010. Our calculations using their rates are shown in black, and they agree with theirs.

explained by SNe Ia and massive stars. The maximum of the contribution occurs at low bulge-to-disk ratios. Uncertainties of this contribution come from the SN Ia Galactic rates (Milne et al. 2004). Such an incomplete attribution to SNe Ia of the $511 \mathrm{keV}$ line has led to the suggestion that most of the contribution comes from annihilation of dark matter (Boehm et al. 2004; Fayet 2004). In the GeV regime, the lack of a full contribution from astrophysical sources such as blazars led to the proposal that weakly interacting massive particle (WIMP) annhilations in the Galactic halo could be the main contributor to the diffuse $\mathrm{GeV}$ background fluxes. Those WIMPs would have masses in the range from 0.1 to $10 \mathrm{GeV}$, for a variety of dark matter halo models (Pullen et al. 2007). However, recent work (Ajello et al. 2015; Di Mauro \& Donato 2015) greatly constrains the dark matter contributions in this energy range. These authors find that blazars, together with star-forming and radio galaxies, can account for the gamma-ray background there.

In a similar way, the early estimates of SN Ia rates at high $z$ (D04) predicted a small contribution from $\mathrm{SNe}$ Ia to the gamma-ray background (AKH05). In that calculation, the SN Ia contribution is so small that even the $2 \sigma$ upper bound derived from the D04 SN Ia rates cannot account for the measured gamma-ray background (AKH05, and Figure 7 of this work). This opened a window for contributions from WIMP decays in the $\mathrm{MeV}$ range (see, e.g., AKH05; Cembranos et al. 2007), or from new populations of quasars (Ajello et al. 2009), among others. Here we show that the recent estimates of SN Ia rates at high $z$, taken at face value, cannot provide an explanation for the cosmic background in the $\mathrm{MeV}$ range.

Recently, Ajello et al. (2008), analyzing the $3 \mathrm{yr}$ data from the Swift/Burst Alert Telescope survey, concluded that the flatspectrum radio quasars (FSRQs) could account for most, or even all, of the cosmic gamma-ray background for energies 


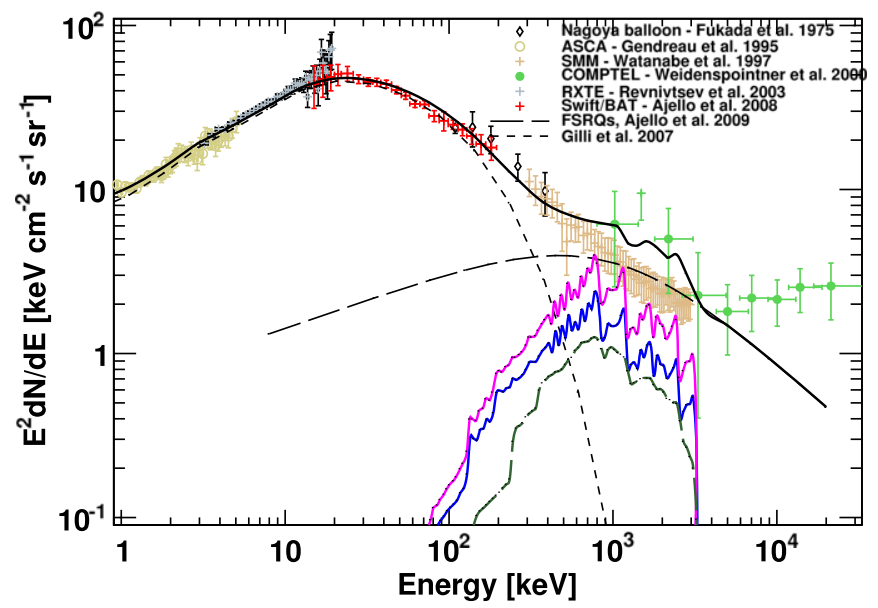

Figure 13. Cosmic gamma-ray background in the $1-30,000 \mathrm{keV}$ range, from different sources. Shown as the dot-dashed dark-green line is the predicted contribution by SNe Ia, when adopting the $2 \sigma$ upper limit of the SN Ia rates from CANDELS (Rodney et al. 2014), while in blue is that corresponding to the $1 \sigma$ upper bound of the rates by Okumura et al. (2014), and in magenta we show the gamma-ray background from the upper $2 \sigma$ to the rates from Subaru/ $X M M-N e w t o n$ (Okumura et al. 2014), the magenta line ( $2 \sigma$ upper bound) being very unrealistic. The long-dashed black line is the contribution from FSRQs (Ajello et al. 2009), while the solid black line is the total gamma-ray background of the long-dashed black line (FSRQs) and background from the $1 \sigma$ upper bound of $\mathrm{SN}$ Ia rates from Subaru/XMM-Newton (Okumura et al. 2014). This gives a good fit to the observed gamma-ray background of SMM (Watanabe et al. 1997) but only because of the FSRQ contribution.

above $0.5 \mathrm{MeV}$ by assuming that its inverse Compton peak is located in the $\mathrm{MeV}$ band. We show in Figure 13 as the longdashed black line the best estimate of the contribution of FSRQs to the cosmic gamma-ray background. In Figure 13, the solid black line is the total contribution of FSRQs and SNe Ia when we adopt the $1 \sigma$ upper bound of SN Ia rates from Subaru/XMM-Newton (Okumura et al. 2014). The decline of the FSRQs that match the obeservations above $10 \mathrm{MeV}$ is $d N /$ $d E \sim E^{-2.5}$. The shape of the SN Ia contribution is very similar up to $3 \mathrm{MeV}$. Clearly, the gamma-ray background from $\mathrm{SNe} \mathrm{Ia}$ can improve the fit of FSRQs to the measured gamma-ray background between 0.3 and $0.8 \mathrm{MeV}$.

\section{CONCLUSIONS}

Our calculations demonstrated that the use of SN Ia rates "as measured" at various redshifts is too low to account for the bulk of the observed gamma-ray background in the $\mathrm{MeV}$ range. We find that, above $z=2-2.5$, the gamma-ray emission from $\mathrm{SNe}$ Ia makes no significant contribution to the cosmic background. It is thus not expected that future determinations of the SN Ia rates above that range would make a noticeable difference. However, what does make a difference is the overall $\mathrm{SN}$ Ia rate from $z=0$ to $z=2$. We have shown here that recently reported values of the SN Ia rates from Okumura et al. (2014), Graur et al. (2014), and Rodney et al. (2014) predict a contribution that is about a factor of 5 below the observed background. Contributions from $z$ beyond 2 are, as noted, too distant to be significant, even in the most optimistic cases, where rates continue to grow (see Figures 5 and 6).

A relevant aspect of the uncertainties in determining rates at high $z(>1.4)$ is that the detection efficiency decreases rapidly with redshift, as observed bands shift farther into the rest-frame UV spectrum, where SN Ia emission is scarce (see the spectra shown in Riess et al. 2007). This causes the uncertainties to become very large at $z \sim 1.6$ (Barbary et al. 2012). This question and the one of addressing dust obscuration, which requires a model for cosmic dust evolution, make the measurements of the rates at high $z$ very unreliable. Note, however, the good agreement between the plethora of measurements of SNe Ia at low $z$ (Figure 6).

The current measurements of rates at high $z$ concentrate on normal (cosmological) SNe Ia. They make up to about $60 \%$ of all SN Ia explosions (see Ruiz-Lapuente 2014, for a review). The average predictions for the gamma-ray background, which at face value give gamma-ray backgrounds that are too low, might slightly change if the bulk of the noncosmological SNe Ia were included. It has been estimated that up to $30 \%$ of SNe Ia could be of the Type Iax (Foley et al. 2013), though a lower estimate is found in White et al. (2015). Those SNe Ia produce smaller amounts of ${ }^{56} \mathrm{Ni}$, thus making an even smaller contribution to the background (although their smaller ejecta mass increases the gamma-ray escape fraction). Furthermore, there is also a $15 \%$ group of SN $1991 \mathrm{bg}$-like events, which are much less luminous than the cosmological SNe Ia.

From the opposite perspective, a factor that could make the mean gamma-ray fluxes larger would be to adopt models brighter in gamma rays than W7 or W7fm (both models giving about the same average contribution over 600 days after explosion); some SN Ia models can give twice as many escaping gamma rays. One should acknowledge, however, that those models might fare worse in the comparison with observed spectra and light curves in the optical range. The first SN Ia observed in gamma rays, SN 2014J (Churazov et al. 2014; Diehl et al. 2014), can be well reproduced within the set of realistic models presented in this paper. SN 2014J synthesized an amount of ${ }^{56} \mathrm{Ni}$ that falls within the standard range (about $0.5-0.6 M_{\odot}$ ). That $\mathrm{SN}$ is best explained as the explosion of a WD close to the Chandrasekhar mass (Diehl et al. 2014). Having considered a wide range of models, we find that this is not the decisive point with regard to the question we address in the paper, i.e., whether SNe Ia are major contributors to the gamma-ray background in the $\mathrm{MeV}$ range.

In summary, following many measurements and estimates of the SN Ia rate as a function of redshift, we now have a more reliable estimate of the SN Ia induced gamma-ray background in the $\mathrm{MeV}$ range than was possible at the end of the twentieth century and beginning of the twenty-first. As the yields of different SN Ia models do not vary greatly, the conclusions are nearly independent of the particular model taken to represent normal SNe Ia. Attributing the gamma-ray background to $\mathrm{SNe}$ Ia appears tempting, as we know that they are significant gamma-ray sources, but within the current uncertainties SNe Ia do not seem able to account for all of the $\mathrm{MeV}$ background. One needs to look for another source of the gamma-ray background in the $\mathrm{MeV}$ range, with FSQRs as a promising object class. SNe Ia can produce up to $30 \%-50 \%$ of the gamma-ray background emission (if we take observed event rates at face value), and the remaining one-half to two-thirds would have to be attributed to one or more contributors.

P.L.R. would like to thank the Max-Planck-Institut für Astrophysik, where this project was started. L.S.T. would like to thank the Palmetto Cluster administrative and support team of Clemson University for giving us the computational time to finish this work. The authors also thank Jun Okumura and Steve Rodney for useful information concerning the observed 
$\mathrm{SN}$ rates. We thank as well Shunsaku Horiuchi and John Beacom for providing their predictions for rates and background. Our thanks go as well to Ivo Seitenzahl and Rüdiger Pakmor for providing data of their SN Ia explosion models. P.R.-L. and R.C. acknowledge support from grant AYA201236353, of the Ministerio de Economía y Competitividad (MINECO) of Spain. F.K.R. receives support from the Emmy Noether Program (RO 3676/1-1) of DFG and the ARCHES prize of the German Federal Ministry of Education and Research (BMBF). S.T.O. acknowledges financial support from Studienstiftung des deutschen Volkes.

\section{REFERENCES}

Ade, P. A. R., Aghanim, N., Armitage-Caplan, C., et al. 2014, A\&A, 571, A16

Ahn, K., Komatsu, E., \& Höflich, P. 2005, PhRvD, 71, 021303 (AKH05)

Ajello, M., Costamante, L., Sambruna, R., et al. 2009, ApJ, 699, 603

Ajello, M., Gasparrini, D., Sánchez-Conde, M., et al. 2015, ApJL, 800, L27

Ajello, M., Greiner, J., Sato, G., et al. 2008, ApJ, 689, 666

Ambwani, K., \& Sutherland, P. 1998, ApJ, 325, 820

Barbary, K., Aldering, G., Amanullah, R., et al. 2012, ApJ, 745, 31

Blanc, G., Afonso, C., Alard, C., et al. 2004, A\&A, 423, 881

Boehm, C., Hooper, D., Silk, J., Cassé, M., \& Paul, J. 2004, PhRvL, 92 101301

Botticella, M. T., Riello, M., Cappellaro, E., et al. 2008, A\&A, 479, 49

Burrows, A., \& The, L.-S. 1990, ApJ, 360, 626

Bussard, R. W., Ramaty, R., \& Drachman, R. J. 1979, ApJ, 228, 928

Cappellaro, E., Evans, R., \& Turatto, M. 1999, A\&A, 351, 459

Cembranos, J. A. R., Feng, J. L., \& Strigari, L. E. 2007, PhRvL, 99, 1301

Churazov, E., Sunyaev, R., Isern, J., et al. 2014, Natur, 512, 406

Ciaraldi-Schoolmann, F., Seitenzahl, I. R., \& Röpke, F. K. 2013, A\&A, 559, A117

Clayton, D. D., \& Silk, J. 1969, ApJL, 158, L43

Clayton, D. D., \& The, L.-S. 1991, ApJ, 375, 221

Dahlen, T., Strolger, L.-G., \& Reiss, A. G. 2008, ApJ, 681, 462

Dahlen, T., Strolger, L.-G., Riess, A. G., et al. 2004, ApJ, 613, 189 (D04)

Di Mauro, D., \& Donato, F. 2015, PhRvD, 91, 123001

Diehl, R., Siegert, T, Hillebrandt, W., et al. 2014, Sci, 345, 1162

Dilday, B., Kessler, R., Frieman, J. A., et al. 2008, ApJ, 682, 262

Dilday, B., Smith, M., Bassett, B., et al. 2010, ApJ, 713, 1026

Fayet, P. 2004, PhRvL, 70, 3514

Foley, R. J., Challis, P. J., Chornock, R., et al. 2013, ApJ, 767, 57

Foley, R. J., \& Mandel, K. 2013, ApJ, 778, 167

Fukada, Y., Hayakawa, S., Kasahara, I., et al. 1975, Natur, 254, 398

Graur, D., \& Maoz, D. 2013, MNRAS, 430, 1376

Graur, O., Poznanski, D., Maoz, D., et al. 2011, MNRAS, 417, 916

Graur, O., Rodney, S. A., Maoz, D., et al. 2014, ApJ, 783, 28G

Hardin, D., Afonso, C., Alard, C., et al. 2000, A\&A, 362, 419

Horesh, A., Poznanski, D., Ofek, E. O., \& Maoz, D. 2008, MNRAS, 389, 1871

Horiuchi, S., \& Beacom, J. F. 2010, ApJ, 723, 329

Iwabuchi, K., \& Kumagai, S. 2001, PASJ, 53, 669

Kappadath, S. C., Ryan, J., Bennett, K., et al. 1996, A\&AS, 120, 619
Kinzer, R. L., Jung, G. V., Gruber, D. E., Matteson, J.-L., \& Peterson, L. E. 1997, ApJ, 475, 361

Kuznetsova, N., Barbary, K., Connolly, B., et al. 2008, ApJ, 673, 981

Lacki, B. C., Horiuchi, S., \& Beacom, J. F. 2014, ApJL, 786, L40

Lehoucq, R., Cassé, M., \& Cesarsky, C. 1989, A\&A, 224, L117

Li, W., Chornock, R., Leaman, J., et al. 2011a, MNRAS, 412, 1473

Li, W., Leaman, J., Chornock, R., et al. 2011b, MNRAS, 412, 1441

Madau, P., Ghisellini, G., \& Fabian, A. C. 1994, MNRAS, 270, L17

Melinder, J., Dahlen, T., Mencía Trinchant, L., et al. 2012, A\&A, 545, A96

Milne, P. A., Hungerford, C. L., Fryer, C. L., et al. 2004, ApJ, 613, 1101

Nomoto, K., Thielemann, F.-K., \& Yokoi, K. 1984, ApJ, 286, 664

Okumura, J. E., Ihara, Y., Doi, M., et al. 2014, PASJ, 66, 49

Pain, R., Fabbro, S., Sullivan, M., et al. 2002, ApJ, 577, 120

Pakmor, R., Kromer, M., Taubenberger, S., et al. 2012, ApJL, 747, L10

Perrett, K., Sullivan, M., Conley, A., et al. 2012, AJ, 144, 59

Pullen, A. R., Chary, R.-R., \& Kamionkowski, M. 2007, PhRvD, 76, 3006

Riess, A. G., Strolger, L.-G., Casertano, S., et al. 2007, ApJ, 659, 98

Rodney, S. A., Riess, A. G., Strolger, L.-G., et al. 2014, AJ, 148, 13

Rodney, S. A., \& Tonry, J. L. 2010, ApJ, 723, 47

Röpke, F. K., Kromer, M., Seitenzahl, I. R., et al. 2012, ApJL, 750, L19

Ruiter, A. J., Belczynski, K., Sim, S. A., et al. 2011, MNRAS, 417, 408

Ruiter, A. J., Sim, S. A., Pakmor, R., et al. 2013, MNRAS, 429, 1425

Ruiz-Lapuente, P. 2001, in AIP Conf. Proc. 555, Cosmology and Particle Physics: CAPP 2000, ed. R. Durrer, J. Garcia-Bellido, \& M. Shaposhnikov (Melville, NY: AIP), 575

Ruiz-Lapuente, P. 2014, NewAR, 62, 15

Ruiz-Lapuente, P., Cassé, M., \& Vangioni-Flam, E. 2001, ApJ, 549, 483 (RCV01)

Ruiz-Lapuente, P., Lichti, G. G., Lehoucq, R., Canal, R., \& Cassé, M. 1993, ApJ, 417, 547

Seitenzahl, I. R., Ciaraldi-Schoolmann, F., Röpke, F. K., et al. 2013, MNRAS, 429, 1156

Sim, S. A., Röpke, F. K., Hillebrandt, W., et al. 2010, ApJL, 714, L52

Sreekumar, P., Bertsch, D. L., Dingus, B. L., et al. 1998, ApJ, 494, 523

Strigari, L. E., Beacom, J. F., Walker, T. P., \& Zhang, P. 2005, JCAP, 04, 017 S

Summa, A., Ulyanov, A., Kromer, M., et al. 2013, A\&A, 554, A67

The, L.-S., Bridgman, W. T., \& Clayton, D. D. 1994, ApJS, 93, 531

The, L.-S., \& Burrows, A. 2014, ApJ, 786, 141

The, L.-S., Burrows, A., \& Bussard, R. 1990, ApJ, 352, 73

The, L.-S., Leising, M. D., \& Clayton, D. D. 1993, ApJ, 403, 32

Tonry, J. L., Schmidt, B. P., Barris, B., et al. 2003, ApJ, 594, 1

Trombka, J. I., Dyer, C. S., Evans, L. G., et al. 1977, ApJ, 212, 925

Ueda, Y., Akiyama, M., Ohta, K., \& Miyaji, T. 2003, ApJ, 598, 886

Watanabe, K., Hartmann, D. H., Leising, M. D., et al. 1997, in AIP Conf. Proc. 410, The Fourth Compton Symposium (Melville, NY: AIP), 1223

Watanabe, K., Hartmann, D. H., Leising, M. D., \& The, L.-S. 1999a, ApJ, 516, 285 (W99b)

Watanabe, K., Leising, M., Share, G., \& Kinzer, R. 1999b, BAAS, 31, 702

Weidenspointner, G. 1999, PhD thesis, Technische Univ. München

Weidenspointner, G., Varendorff, M., Kappadath, S. C., et al. 2000, in AIP Conf. Proc. 510, The Fifth Compton Symposium, ed. M. L. McConnell, \& J. M. Ryan (Melville, NY: AIP), 581

White, C. J., Kasliwal, M. M., Nugent, P. E., et al. 2015, ApJ, 799, 52

Yamaoka, H., Nomoto, K., Shigeyama, T., \& Thielemann, F.-K. 1992, ApJL, 393, L55

Zdziarski, A. A. 1996, MNRAS, 281, L9 